\title{
Expression and Prognosis of MYD88 L265p Mutation in Diffuse Large B-Cell Lymphoma
}

\author{
Yiming Zhao, Rui Zhang, Ling Ge, Wei Qian, Fengbo Jin, Mingzhen Yang* \\ Department of Hematology, The Fourth Affiliated Hospital of Anhui Medical University, Hefei, China \\ Email: *ymz89@163.com
}

How to cite this paper: Zhao, Y.M., Zhang, R., Ge, L., Qian, W., Jin, F.B. and Yang, M.Z. (2020) Expression and Prognosis of MYD88 L265p Mutation in Diffuse Large B-Cell Lymphoma. Open Journal of Blood Diseases, 10, 133-139. https://doi.org/10.4236/ojbd.2020.104015

Received: November 10, 2020

Accepted: December 26, 2020

Published: December 29, 2020

Copyright $\odot 2020$ by author(s) and Scientific Research Publishing Inc. This work is licensed under the Creative Commons Attribution International License (CC BY 4.0).

http://creativecommons.org/licenses/by/4.0/ (c) (i) Open Access

\begin{abstract}
Objective: To detect the positive rate of MyD88 1265p mutation in DLBCL patients, and analyze the correlation between MYD88 L265p mutation and DLBCL prognosis. Methods: In this study, 40 formalin fixed paraffin embedded tissue samples were collected from the pathology department of the Fourth Affiliated Hospital and the First Affiliated Hospital of Anhui Medical University from January 2017 to December 2019. The relationship between MYD88 L265p gene mutation and clinicopathological parameters of DLBCL, such as age, gender, Ann Arbor stage, site, B symptom, NCCN-IPI and IPI score, ECOG PS score, serum ferritin, $\beta 2$-microglobulin and LDH levels were analyzed. Results: The positive rate of MYD88 L265p was 20\% (8/40), 25.0\% $(6 / 24)$ in non GCB and $12.5 \%(2 / 16)$ in GCB. There were no significant differences in age, gender, location, extranodal invasion site, $\beta 2$-microglobulin, serum ferritin and LDH between wild-type and mutant type of MYD88 gene, but had statistical significance with Han's classification. MYD88 L265p gene mutation was associated with Ann Arbor stage, NCCN-IPI score and IPI score. Conclusion: MYD88 L265P mutation is closely related to the occurrence, development and prognosis of DLBCL. It is an adverse prognostic factor of DLBCL and may be used for the prognosis evaluation of DLBCL.
\end{abstract}

\section{Keywords}

DLBCL, MYD88 L265P, PCR, Prognosis

\section{Introduction}

Diffuse large B cell lymphoma is the most common type of non-Hodgkin lymphoma with an incidence rate of about $1 / 3$ in NHL [1]. The median age of onset of DLBCL was more than 60 years old. According to different gene expression patterns, DLBCL was divided into germinal center B-cell like lymphoma (GCB), 
activated B-cell lymphoma $(\mathrm{ABC})$ and primary mediastinal diffuse large $\mathrm{B}$-cell lymphoma (PMBL) [2]. At present, the first-line treatment of DLBCL mainly adopts R-CHOP Regimen. However, studies have shown that GCB DLBCL has a better overall survival period while ABC type DLBCL has a poor prognosis, and the total effective rate is less than $40 \%$ [3]. The international prognostic index (IPI) score is based on the number of extranodal involvement sites, Ann Arbor stage, age, lactate dehydrogenase, $\beta 2$-microglobulin, ECOG PS score, as risk factors for prognosis. However, IPI risk stratification score is not the only tool to evaluate the prognosis of patients. Patients with the same IPI score have different survival outcomes, as well as non GCB subtype, double expression of DLBCL (c-myc, bcl-2 expression at the same time), poor response to standard R-CHOP chemotherapy and poor prognosis. Finding new molecular markers related to prognosis will be helpful for better diagnosis and treatment of patients with poor prognosis of DLBCL.

In recent years, with the development of high-throughput technologies such as total exon sequencing and deep sequencing, people have a deeper understanding of the pathogenesis and disease susceptibility of DLBCL. Some studies have found that there is a functional activation of myeloid differentiation factor 88 (MYD88) 1265p gene mutation in a variety of B-cell tumors (that is, a T-C mutation in the 794 base of MyD88 coding sequence, resulting in a missense mutation of leucine at 265 in the coding region of MyD88 to proline). This mutation leads to abnormal activation of the signal pathway, suggesting that MYD88 is involved in a variety of B-cell tumors. The abnormality of DLBCL may be related to the occurrence, development and prognosis of DLBCL [4]. Therefore, it is necessary to further explore the effect of MYD88 L265P mutation on the occurrence and development of DLBCL. This study detected the expression of MYD88 L265P mutation in newly diagnosed patients with DLBCL, and analyzed its impact on the prognosis of patients with DLBCL, which further improved the clinical understanding of DLBCL. The analysis is as follows.

\section{Material and Methods}

\subsection{General Information}

In this study, 40 formalin fixed paraffin embedded tissue samples were collected from the pathology department of the Fourth Affiliated Hospital and the First Affiliated Hospital of Anhui Medical University from January 2017 to December 2019. According to the diagnostic criteria of the World Health Organization classification of hematopoiesis and lymphoid tissue tumors in 2016 [5], the diagnosis was made by two senior pathologists in the department. A total of 40 newly diagnosed DLBCL patients, including 18 males and 22 females, aged from 20 to 66 years, with a median age of 37.0 years. At the same time, the clinicopathological data of these patients were further collected, such as age, gender, Ann Arbor stage, site, B symptoms, NCCN-IPI score, ECOG PS score, serum ferritin, $\beta 2$-microglobulin and LDH levels. This study was approved by the ethics com- 
mittee of Anhui Medical University.

\subsection{MYD88 L265p Mutation Detection}

Formalin fixed paraffin embedded tissue samples were collected. DNA was extracted from FFPE tissue section. According to reference [6], MYD88 L265P mutation was detected by real-time as PCR. FastPure FFPE DNA Isolation Kit was used to separate and purificate genomic DNA from formalin-fixed paraffin-embedded (FFPE) tissues, High-quality DNA can be obtained by a centrifugal adsorption column that specifically binds DNA and a unique buffer system. The extracted genome with good integrity, high purity, stable and reliable quality can be used in various downstream applications including PCR, qPCR, library construction. The primers are 5'-GTGCCCATCAGAAGCGCCT-3' (wild type), 5'-GTGCCCATCAGAAGCG CCC-3' (mutant), and 5'-GACGTGTCTGT GAAGTTGGCATCTC-3' (reverse). The manufactures information of analytical instruments is Illumina Hiseq-X High throughput sequencer, Illumina cbot Cluster Station, Illumina Genome Analyzer IIx, Invitrogen Qbit Spectrophotometer and EPPENDORF Single channel adjustable pipette.

\subsection{Statistical Analysis}

Chi square test and Fisher exact test were used to analyze the relationship between MYD88 L265P and clinicopathological features of DLBCL patients. All data were processed and analyzed by spss19.0 statistical software.

\section{Result}

\subsection{Incidence of MYD88 L265P Mutation in Diffuse Large B-Cell Lymphoma}

According to Hans classification, 16 cases $(40.0 \%, 16 / 40)$ belonged to GCB type, 24 cases $(60.0 \%, 24 / 40)$ belonged to non-GCB type, GCB: non-GCB was about 1:1.5. The positive rate of MYD88 L265P was 20\% (8/40), 25.0\% (6/24) in non-GCB and $12.5 \%(2 / 16)$ in GCB.

\subsection{Relationship between MYD88 L265P Mutation and Clinical Data in Diffuse Large B-Cell Lymphoma}

This study also analyzed the relationship between MYD88 L265P mutation and clinical data in diffuse large B-cell lymphoma. The results showed that there were no significant differences in age, gender, location, extranodal invasion site, $\beta 2$-microglobulin, ferritin and LDH between wild-type and mutant type of MYD88 L265P gene $(\mathrm{P}>0.05)$, and had statistical significance with Han's classification $(\mathrm{P}<0.05)$ (see Table 1$)$.

\subsection{Relationship between MYD88 L265P Gene Mutation and Prognosis of DLBCL}

In order to analyze the relationship between MYD88 L265P gene mutation and 
prognosis of DLBCL, DLBCL patients were grouped according to different grouping methods, and the occurrence of MYD88 L265P gene mutation in different groups was analyzed. The results showed that there were significant differences between MYD88 L265P 1265p gene mutation classification and Ann Arbor stage, NCCN-IPI score and IPI score $(\mathrm{P}<0.05)$ (see Table 2$)$.

Table 1. Relationship between MYD88 L265P mutation and clinical data in diffuse large B-cell lymphoma.

\begin{tabular}{|c|c|c|c|}
\hline \multirow{2}{*}{ Clinicopathological parameters } & \multicolumn{2}{|c|}{ MYD88 L265P gene mutation } & \multirow{2}{*}{$P$ value } \\
\hline & Wild type $(\mathrm{N}=32)$ & Mutant type $(\mathrm{N}=8)$ & \\
\hline Age & & & 1.000 \\
\hline$<60$ & 18 & 5 & \\
\hline$\geq 60$ & 14 & 3 & \\
\hline Gender & & & 0.529 \\
\hline Male & 15 & 3 & \\
\hline Female & 17 & 5 & \\
\hline Site & & & 0.079 \\
\hline Intranodal & 20 & 1 & \\
\hline Extranodal & 12 & 7 & \\
\hline Extranodal invasion site & & & 0.195 \\
\hline $\begin{array}{c}\text { Central nervous } \\
\text { system involvement }\end{array}$ & 6 & 5 & \\
\hline $\begin{array}{l}\text { Other extranodal } \\
\text { violations }\end{array}$ & 6 & 2 & \\
\hline Hans' classification & & & 0.014 \\
\hline GCB & 17 & 1 & \\
\hline Non-GCB & 15 & 7 & \\
\hline Serum ferritin & & & 0.872 \\
\hline Normal & 18 & 4 & \\
\hline High & 14 & 4 & \\
\hline$\beta 2$-microglobulin & & & 0.174 \\
\hline Normal & 13 & 4 & \\
\hline High & 19 & 4 & \\
\hline LDH & & & 1.000 \\
\hline Normal & 17 & 5 & \\
\hline High & 15 & 3 & \\
\hline
\end{tabular}


Table 2. Relationship between MYD88 L265P gene mutation and prognosis of DLBCL.

\begin{tabular}{|c|c|c|c|}
\hline \multirow{2}{*}{ Clinicopathological parameters } & \multicolumn{2}{|c|}{ MYD88 L265P gene mutation } & \multirow{2}{*}{$P$ value } \\
\hline & Wild type $(\mathrm{N}=32)$ & Mutant type $(\mathrm{N}=8)$ & \\
\hline Ann Arbor staging & & & 0.042 \\
\hline I-II & 12 & 1 & \\
\hline III-IV & 20 & 7 & \\
\hline B symptoms & & & 1.000 \\
\hline yes & 11 & 3 & \\
\hline no & 21 & 5 & \\
\hline ECOG PS score & & & 0.202 \\
\hline$<2$ & 23 & 5 & \\
\hline$\geq 2$ & 9 & 3 & \\
\hline IPI score & & & 0.039 \\
\hline Low risk & 5 & 0 & \\
\hline Medium-low risk & 13 & 0 & \\
\hline Medium-high risk & 11 & 4 & \\
\hline Extremely high risk & 3 & 4 & \\
\hline NCCN-IPI score & & & 0.041 \\
\hline Low risk & 6 & 0 & \\
\hline Medium-low & 13 & 2 & \\
\hline Medium-high & 10 & 4 & \\
\hline Extremely high risk & 3 & 2 & \\
\hline
\end{tabular}

\section{Discussion}

MYD88, a soluble adaptor protein in cytoplasm, is a member of the toll like receptors (TLR)/interleukin-1 receptor (IL-1R) family and death domain family, which mediates the signal transduction of most TLR, IL-1R and IL-18R cells. Furthermore, it can activate transcription factors such as NF - $\kappa$ B and cause the release of a variety of inflammatory cytokines and anti apoptotic molecules, and participate in human innate immunity [7]. MYD88 L265p deficient mice are susceptible to a variety of microorganisms, while in humans, MYD88 L265p function loss is prone to fatal suppurative bacterial infection. Therefore, it is considered that the over activation of this pathway is related to autoimmune diseases [8]. In recent years, missense mutation of amino acid 265 in coding region of MYD88 protein has been found in many B cell tumors, that is, leucine to proline (MYD88 L265p). It has been reported that 29\% of ABC type diffuse large B-cell lymphoma has MYD88 L265p [9]. The total positive rate of MYD88 L265p was $20 \%$ in this study. The positive rate of non GCB and GCB was $25.0 \%$ and $12.5 \%$ respectively. 
DLBCL is a highly heterogeneous lymphoma. The standard treatment for most patients is R-CHOP. The 3-year event free survival rate is about $60 \%$. However, the response rates of different DLBCL subtypes to chemotherapy are different. In addition, more than $1 / 3$ of patients with remission after chemotherapy will relapse and enter the stage of tumor progression, which may be related to the individual differences of patients and the drug resistance of tumor cells under the stimulation of chemotherapy drugs. In recent years, studies have found that MYD88 L265p mutation is also closely related to the prognosis of DLBCL. It is reported that the mutation rate is high in ABC type DLBCL and the prognosis is poor, which may be related to the abnormal activation of MyD88 is the key core protein of NF - $\kappa$ B pathway, and the activation of NF - $\kappa$ B pathway only maintains the activity of ABC type DLBCL cells, but not GCB type cells [10].

In this study, MYD88 L265p mutation was positively correlated with Ann Arbor stage, NCCN-IPI score and IPI score. The mutation rate of patients with high score was significantly higher than that of patients with low score, which further confirms that the mutation will affect the prognosis of patients. The results of a meta-analysis of 40 literatures on MYD88 L265p gene mutation showed that the gene mutation was related to age, prognosis and immune classification, but not to clinical stage and gender [11]. The results of this study showed that MYD88 L265p gene mutation was associated with Hans' classification. However, the main limitation of this study is the small sample size, which needs to be further verified in a larger cohort.

\section{Conclusion}

In conclusion, MYD88 L265P mutation is associated with Ann Arbor stage, NCCN-IPI score, IPI score and Hans' classification, suggesting that MYD88 L265p mutation is closely related to the occurrence, development and prognosis of DLBCL. It is an adverse prognostic factor of DLBCL and can be used for the prognosis evaluation of DLBCL. MYD88 L265p gene mutation is a key molecule in TLR signal transduction pathway. At present, there are many drugs targeting MYD88 L265p gene mutation. However, a large number of studies are needed to prove its feasibility, safety and effectiveness in clinical application.

\section{Consent}

Written informed consent was obtained from the patient for publication of this Case report and any accompanying images. A copy of the written consent is available for review by the Editor of this journal.

\section{Funding}

This work is funded by the Research Fund Project of Anhui Medical University (Project No: 2017xkj052). The authors thank the patient and his family and all the investigators, including the physicians and laboratory technicians in this study. 


\section{Conflicts of Interest}

The authors declare no conflicts of interest regarding the publication of this paper.

\section{References}

[1] Flodr, P., Latalova, P., Tichy, M., et al. (2014) Diffuse Large B-Cell Lymphoma: The History, Current View and New Perspectives. Neoplasma, 61, 491-504. https://doi.org/10.4149/neo_2014_062

[2] Rosenwald, A., Wright, G., Chan, W.C., et al. (2002) The Use of Molecular Profiling to Predict Survival after Chemotherapy for Diffuse Large-B-Cell Lymphoma. New England Journal of Medicine, 346, 1937-1947.

[3] Alizadeh, A.A., Eisen, M.B., Davis, R.E., et al. (2000) Distinct Types of Diffuse Large B-Cell Lymphoma Identified by Gene Expression Profiling. Nature, 403, 503-511. https://doi.org/10.1038/35000501

[4] Jardin, F. (2014) Next Generation Sequencing and the Management of Diffuse Large B-Cell Lymphoma: From Whole Exome Analysis to Targeted Therapy. Discovery Medicine, 18, 51-65.

[5] Arber, D.A., Orazi, A., Hasserjian, R., et al. (2016) The 2016 Revision to the World Health Organization Classification of Myeloid Neoplasms and Acute Leukemia. Blood, 127, 2391-2405. https://doi.org/10.1182/blood-2016-03-643544

[6] Xu, L., Hunter, Z.R., Yang, G., et al. (2013) MYD88 L265P in Waldenström Macroglobulinemia, Immunoglobulin M Monoclonal Gammopathy, and Other B-Cell Lymph Proliferative Disorders Using Conventional and Quantitative Allele-Specific Polymerase Chain Reaction. Blood, 121, 2051-2058. https://doi.org/10.1182/blood-2013-05-502211

[7] Kawai, T. and Akira, S. (2010) The Role of Pattern-Recognition Receptors in Innate Immunity: Update on Toll-Like Receptors. Nature Immunology, 11, 373-384. https://doi.org/10.1038/ni.1863

[8] von Bernuth, H., Picard, C., Jin, Z.B., et al. (2008) Pyogenic Bacterial Infections in Humans with MyD88 Deficiency. Science, 321, 691-696. https://doi.org/10.1126/science.1158298

[9] Ngo, V.N., Young, R.M., Schmitz, R., et al. (2011) Oncogenically Active MYD88 Mutations in Human Lymphoma. Nature, 470, 115-119.

[10] Hans, C.P., Weisenburger, D.D., Greiner, T.C., et al. (2004) Confirmation of the Molecular Classification of Diffuse Large B-Cell Lymphoma by Immunohistochemistry Using a Tissue Microarray. Blood, 103, 275-282. https://doi.org/10.1182/blood-2003-05-1545

[11] Lee, J.-H., Jeong, H., Choi, J.-W., et al. (2017) Clinicopathologic Significance of MYD88 L265P Mutation in Diffuse Large B-Cell Lymphoma: A Meta-Analysis. Scientific Reports, 7, 1785. https://doi.org/10.1038/s41598-017-01998-5 